\title{
Características de las tesis de pre-grado de Medicina de una universidad pública del Perú
}

\author{
Betty Castro-Maldonado1,2,a, Claudia Carolina Callirgos-Lozada1,2,a, Martin Kelwin Caicedo-Písfill,2,a, Esteban \\ Alberto Plasencia-Dueñas ${ }^{1,2, a}$, Cristian Díaz-Vélez ${ }^{1,3, b}$.
}

\section{RESUMEN}

Las tesis de pre-grado más que un requisito para titularse son una forma de hacer investigación. Estudios anteriores muestran una publicación de tesis entre $2,7 \%$ a $17,6 \%$.

Objetivo: Describir las características de las tesis de pregrado de una Facultad de Medicina

Material y Métodos: Estudio bibliométrico. Se revisaron 221 tesis, recolectando: Año, número de autores, asesores y referencias bibliográficas, población estudiada y prioridades nacionales de investigación en salud. Se realizó una búsqueda en Google Académico, para evaluar publicación. Se utilizó estadística descriptiva.

Resultados: Del total de tesis, el 91,6\% tuvieron un solo asesor, 76\% fueron descriptivas, $82,8 \%$ se realizó en población hospitalaria y el 62,4\% fue en adultos. Se observó una menor producción en los años 2008-2010. De las tesis entre 2010-2014, $72,9 \%$ no correspondieron a ninguna prioridad nacional de investigación en salud.Sólo el 6,8\% se basó en literatura de los últimos 5 años. Sólo $9(4,1 \%)$ fueron publicadas en una revista científica indizada.

Conclusión: Las tesis de pregrado se caracterizaron por ser descriptivas, hospitalarias, en adultos, basadas en literatura no actualizada y no acordes con las prioridades de investigación en salud. La publicación fué baja. (Horiz Med 2015; 15(3): 34-39)

Palabras clave: Tesis Académica, Educación en pregrado en medicina, Bibliometría. (Fuente: DeCS BIREME).

\section{Characteristics of undergraduate medical thesis of a Peruvian public university}

\section{ABSTRACT}

The undergraduate thesis rather than a requirement to graduate, are a way of doing research. Previous studies show a thesis published between $2.7 \%$ to $17.6 \%$ in indexed journals.

Objective: To describe the characteristics of the undergraduate thesis of a medical school.

Material and Methods: Bibliometric study. 221 theses were reviewed, collecting: year, number of authors, advisers, and references, study population, and national priorities for health research. A search was performed on Google Scholar to assess publication. Descriptive statistics were used.

Results: Of the total, $91.6 \%$ of thesis had one advisor, $76 \%$ were descriptive, $82.8 \%$ were done in a hospital population, $62.4 \%$ in adults. A lower production was found in the years 2008-2010.0f the theses between $2010-2014,72.9 \%$ did not correspond to any national health research priority. Only $6.8 \%$ was based on literature of the last five years. Only $9(4.1 \%)$ were published in anindexed scientific journal.

Conclusion: The undergraduate thesis were characterized as descriptive, in hospitals, in adults, based on out dated literature and not in line with the national priorities for health research. The publication is low. (Horiz Med 2015; 15(3): 34-39)

Key words: Academic Dissertations; Undergraduate Medical Education; Bibliometrics. (Source: MeSH NLM).

Universidad Nacional Pedro Ruiz Gallo. Lambayeque-Perú.

Sociedad Científica de estudiantes de medicina de la Universidad Nacional Pedro Ruiz Gallo. Chiclayo-Perú.

Hospital Nacional Almanzor Aguinaga Asenjo. Chiclayo-Perú. 


\section{INTRODUCCIÓN}

Una opción para obtener el título de médico cirujano en el Perú es con la aprobación de las tesis de pre-grado, lo que obliga indirectamente a los estudiantes a realizar investigación en medicina humana.

La producción científica en América Latina representa menos del $1 \%$ a nivel mundial. Del total de los artículos de América Latina, el Perú aporta el $0,95 \%$ y dentro este pequeño porcentaje encontramos a la investigación médica (1).

En lo que respecta a la participación estudiantil en investigación, solo en $4,5 \%$ de publicaciones de las revistas indizadas a Scielo-Perú hubo participación de un estudiante (1).

Estudios realizados en la Universidad de San Martin de Porres, Universidad Nacional Mayor de San Marcos, Universidad Peruana Cayetano Heredia encontraron que el porcentaje de tesis de pregrado publicadas fue $2.7 \%, 11.8 \%$ y $17.6 \%$ respectivamente $(1,2,3)$.

Según Decreto Legislativo 739 del año 1991, se aprobó el bachillerato automático, obteniéndose el título profesional mediante las siguientes modalidades: presentación y aprobación de la tesis; o cualquier otra modalidad que estime conveniente la Universidad. Basado en este decreto la FMH-UNPRG implementó la modalidad de curso de titulación durante los años 2008-2012 (4).

El objetivo planteado para este estudio fué describir las características de las tesis de pregrado de la Facultad de Medicina Humana de la Universidad Nacional Pedro Ruiz Gallo en el periodo 2006-2014.

\section{MATERIAL Y MÉTODOS}

Se realizó un estudio bibliométrico durante los meses de mayo-junio del 2015 en la biblioteca del Departamento de Ciencias Clínicas y en la oficina del Centro de Investigación de la FMH-UNPRG. La población de estudio fueron las tesis de pregrado de la FMH-UNPRG presentadas en el periodo 20062014, las cuales sumaron un total de 229. De éstas, 8 de los in extensos no se hallaron disponibles por diversos motivos, por lo que el número final de tesis incluidas en el estudio fue de 221. No se realizó cálculo de tamaño de muestra ni muestreo, puesto que se trabajó con todo el universo muestral disponible.

Una vez obtenidos los in extensos, se procedió a recolectar los siguientes datos: Año de presentación, título de la tesis, número de autores, número de asesores, número de jurados, población estudiada (lugar dónde se obtuvo, grupo etáreo), tipo de estudio, lugar de ejecución, número de referencias bibliográficas, número de referencias que especifican año y número de referencias de los últimos 5 años. Puesto que el Ministerio de Salud estableció las Prioridades Nacionales de Investigación en Salud para los años 2010-2014, se evaluó cuántas de las tesis presentadas en el periodo, correspondían a dichas prioridades.

Se realizó una búsqueda en Google Scholar, utilizando el algoritmo de búsqueda propuesto por MaytaTristán y Mezones-Holguín (5), el cual consiste en ingresar el título completo al buscador de Google Académico; si no se encuentran coincidencias se ingresa tres palabras clave (referidas a variables principales, lugar y suj etos de estudio/ población) en combinación con el apellido del primer, segundo y último autor utilizando las herramientas de búsqueda avanzada; de no obtener resultados se repite la búsqueda pero en idioma inglés, con el fin de ver cuántas de las tesis recolectadas han sido publicadas.

Se realizó estadística descriptiva con el programa Microsoft Excel, utilizando frecuencias y proporciones, para las variables número de asesores, tipo de estudio, lugar ejecución, tipo población, grupo etáreo, prioridad y publicación. Para la variable número de referencias bibliográficas, y referencia bibliográfica en los últimos 5 años se calcularon medias y desviación estándar.

Se creó la variable porcentaje de referencias bibliográficas actuales (se obtuvo dividiendo el número de referencia bibliográficas en los últimos 
5 años entre el total de referencias bibliográficas de cada tesis)y se categorizó en 4 grupos: $\leq 25 \%$, 25 $50 \%, 50-75 \%,>75 \%$.

\section{RESULTADOS}

De un total de 229 tesis de pregrado presentadas entre los años 2006-2014, se contaron con 221 tesis in-extenso, las cuales fueron revisadas, observándose una tendencia muy irregular en su producción, llegándose a encontrar 48 tesis en el 2006, hallándose una menor producción en los años 2008-2010 (6, 8, 5 tesis respectivamente). Figura 1.

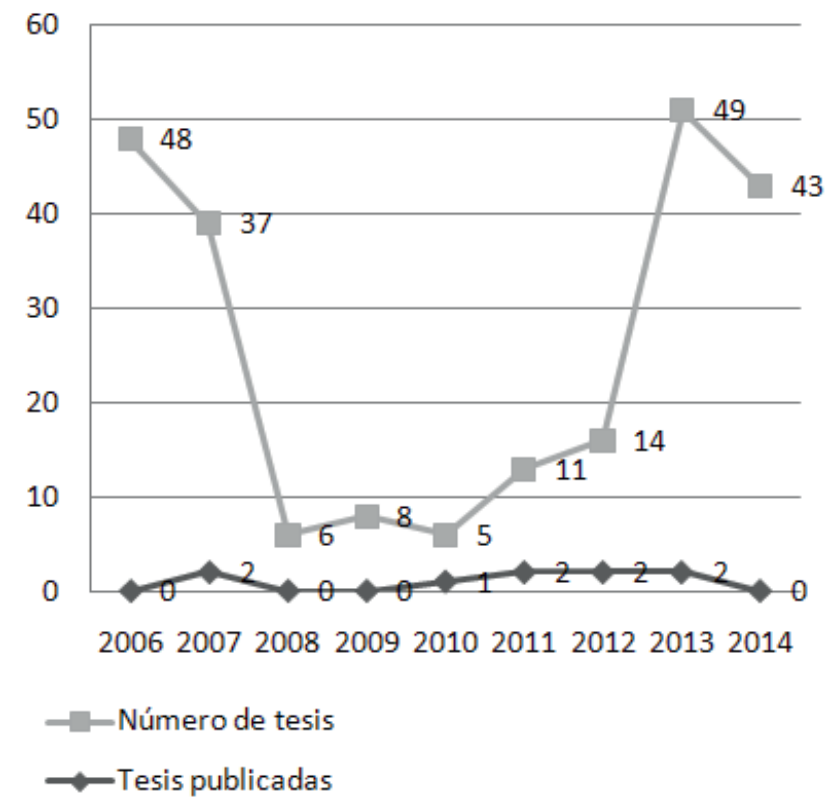

Figura 1. Tesis de Medicina Humana de la UNPRG sustentadas y publicadas entre 2006-2014

El $91,6 \%$ de las tesis tuvieron un solo asesor, el diseño del estudio fue en el $76 \%$ descriptivo, $82,8 \%$ se realizó en población hospitalaria y $62,4 \%$ en adultos. De las tesis sustentadas entre 2010-2014 el $72,9 \%$ no correspondieron a ninguna prioridad nacional de investigación en salud. Del total de tesis, solo 9 (4,07\%) fueron publicadas en una revista científica indizada. Tabla 1.
Tabla 1. Características de las tesis de pregrado de la FMH-UNPRG. Periodo 2006-2014

\begin{tabular}{|c|c|c|}
\hline Variable & $\mathrm{N}$ & $\%$ \\
\hline \multicolumn{3}{|l|}{ Número de asesores } \\
\hline 0 & 6 & 0,9 \\
\hline 1 & 197 & 91,6 \\
\hline 2 & 12 & 5,6 \\
\hline 3 & 3 & 1,4 \\
\hline 4 & 1 & 0,5 \\
\hline \multicolumn{3}{|l|}{ Tipo de estudio } \\
\hline Descriptivo & 168 & 76 \\
\hline Analíticos & 53 & 24 \\
\hline \multicolumn{3}{|l|}{ Lugar de ejecución } \\
\hline Lambayeque & 187 & 84,6 \\
\hline Lima & 23 & 10,4 \\
\hline Otros & 11 & 4,9 \\
\hline \multicolumn{3}{|l|}{ Tipo de población } \\
\hline Hospitalario & 183 & 82,8 \\
\hline Centro de Salud & 4 & 1,8 \\
\hline Comunidad & 9 & 4,1 \\
\hline Escolar & 11 & 4,9 \\
\hline Universitario & 7 & 3,2 \\
\hline Otros & 7 & 3,2 \\
\hline \multicolumn{3}{|l|}{ Grupo etáreo } \\
\hline neonatos & 43 & 19,5 \\
\hline niño-adolescentes & 21 & 9,5 \\
\hline adulto & 138 & 62,4 \\
\hline adulto y adulto mayor & 8 & 3,6 \\
\hline Adolescente, adulto, adulto mayor & 2 & 0,9 \\
\hline adulto mayor & 6 & 2,7 \\
\hline \multicolumn{3}{|l|}{ Prioridades $(n=122)^{\star}$} \\
\hline Con prioridad & 33 & 27,1 \\
\hline Sin prioridad & 89 & 72,9 \\
\hline \multicolumn{3}{|l|}{ Tipos de prioridad } \\
\hline Recursos Humanos & 9 & 7,4 \\
\hline Salud mental & 3 & 2,5 \\
\hline Desnutrición Infantil & 4 & 3,3 \\
\hline Mortalidad Materna & 14 & 11,5 \\
\hline Enfermedades trasmisibles & 3 & 2,5 \\
\hline \multicolumn{3}{|l|}{ Publicación } \\
\hline si & 9 & 4,1 \\
\hline no & 212 & 95,9 \\
\hline
\end{tabular}

Las características de las tesis publicadas se presentan en la tabla 2. 
Tabla 2. Características de las tesis de pregrado de la FMH-UNPRG publicadas como artículo científico. Periodo 2006-2014.

\begin{tabular}{|c|c|c|}
\hline Variable & $\mathrm{N}$ & $\%$ \\
\hline \multicolumn{3}{|l|}{ Autores } \\
\hline Al menos un estudiante & 9 & 100 \\
\hline Al menos un asesor & 8 & 88.9 \\
\hline Al menos un jurado & 1 & 11.1 \\
\hline \multicolumn{3}{|l|}{ Autor Corresponsal } \\
\hline Estudiante & 4 & 44.4 \\
\hline Médico & 4 & 44.4 \\
\hline No menciona & 1 & 11.1 \\
\hline \multicolumn{3}{|l|}{ Tipo de Artículo } \\
\hline Artículo Original & 8 & 88.9 \\
\hline Comunicación corta & 1 & 11.1 \\
\hline \multicolumn{3}{|l|}{ Revista } \\
\hline Rev. cuerpo méd. HNAAA & 7 & 77.8 \\
\hline Acta Médica Peruana & 1 & 11.1 \\
\hline Dermatología Peruana & 1 & 11.1 \\
\hline \multicolumn{3}{|l|}{ Financiamiento } \\
\hline Menciona & 0 & 0 \\
\hline No menciona & 9 & 100 \\
\hline
\end{tabular}

*IMRD: Introducción, Material y Métodos, Resultados y Discusión.

La mayoría de las tesis $(120 / 221)$ contaban con 25 $50 \%$ de referencias de los últimos 5 años y sólo 14 $(6,3 \%)$ contaban con más del $75 \%$ de referencias de los últimos 5 años. Tabla 3.

Tabla 3. Frecuencia de tesis de pregrado de la FMH-UNPRG con referencias bibliográficas de últimos 5 años. Periodo 2006-2014.

\begin{tabular}{cc}
\hline Porcentaje de referencias de últimos 5 años (\%) & $\begin{array}{c}\text { Frecuencia } \\
\mathrm{n}(\%)\end{array}$ \\
\hline$<25$ & $36(8,14)$ \\
$25-50$ & $120(27,15)$ \\
$>50-75$ & $51(11,54)$ \\
$>75$ & $14(3,17)$ \\
TOTAL & $221(100)$ \\
\hline
\end{tabular}

\section{DISCUSIÓN}

Se encontró una menor producción de tesis en los años 2008-2010 (6, 8, 5 tesis respectivamente) posiblemente por la apertura del curso de titulación como opción alternativa para titularse, poco compromiso de los estudiantes, al poco incentivo, enseñanza inadecuada y falta de actualización por parte de los docentes del área de investigación.

Resultados similares se encontraron en otros estudios realizados en la ciudad de Lima $(1,2)$, que mencionan como posibles factores: la falta de tiempo de los estudiantes, no percibirse capacitados en metodología de investigación ni en redacción científica, que no existe una cultura de publicación, los trámites engorrosos y la falta de apoyo por parte de su universidad.

Un factor asociado a la intención de realizar una tesis es el tener un familiar médico directo, como se menciona en un trabajo realizado en la ciudad de Ica (8).Se podrían obtener mejores resultados si las universidades capacitaran a sus docentes del área de investigación, motivar a los estudiantes a realizar la tesis en su último año de estudios y promover la investigación cerrando los cursos de titulación.

Según León-Jiménez (7), los cursos del área de investigación deben tener una secuencia lógica como por ejemplo la utilizada en la Universidad Católica Santo Toribio de Mogrovejo: Seminario de tesis I (elaboración del proyecto de investigación), Seminario de tesis II (recojo de la información, procesamiento y análisis preliminar) y Seminario de tesis III (redacción científica y elaboración de un artículo científico), los cuales son llevados consecutivamente durante los últimos ciclos de la carrera.

La mayoría de las tesis optaron por un estudio descriptivo, posiblemente por ser éstos más rápidos, menos costosos y de más fácil análisis estadístico. Un resultado similar se encontró en el trabajo realizado por la UNMSM donde $79.6 \%$ de las tesis emplearon este tipo de estudio (1). 
El lugar de ejecución más frecuente fue en un ambiente hospitalario, siendo la población adulta la más estudiada. Esto puede deberse a la facilidad de obtención de datos del hospital, ya que la mayoría de los estudiantes ejecutan sus tesis en el internado.

Del total de tesis realizadas entre los años 20102014 , el $27,1 \%$ tuvo como tema de investigación una prioridad nacional de investigación en salud, siendo la mortalidad materna la prioridad en salud más estudiada, posiblemente debido al desconocimiento de estas prioridades y a la falta de difusión por parte de la facultad de sus líneas de investigación.

Sólo el 6,8\% de las tesis se basaron en bibliografía actualizada. Según un estudio realizado a nivel nacional el $18,7 \%$ de los estudiantes manifestó tener dificultad para realizar búsqueda bibliográfica (6). La orientación en ese aspecto debe ser responsabilidad de los docentes de las cátedras de investigación, de los asesores y jurados quiénes deberían evaluar la calidad de información que el estudiante utiliza para elaboración de sus tesis y en el momento de la calificación.

Del total de tesis, solo $9(4,1 \%)$ fueron publicadas en una revista científica indizada, cifra menor a la reportada por la UNMSM donde el $11,8 \%$ de las tesis de pregrado del periodo 1998-2008 fueron publicadas (1), y en la Universidad Peruana Cayetano Heredia donde se publicó el $17,6 \%$ de tesis de pregrado del periodo 2000-2003 (3).

Esto puede deberse a la falta de conocimiento por parte de los estudiantes, al trámite engorroso, al considerar la publicación algo superficial, al percibir sus tesis como trabaj os de baja calidad y es posible que la inexperiencia de asesores y jurados esté relacionada con la baja publicación de las tesis (2).

Las universidades deberían fomentar una cultura de publicación entre los estudiantes y docentes, reforzando las revistas de la Región, y que la universidad evalúe la producción científica de los asesores y jurados de las tesis de pregrado.
Otro de los factores que dificulta la publicación de las tesis puede ser la necesidad de adaptar la tesis a un formato conciso de publicación (7).Por ello, una propuesta interesante sería la posibilidad de que los alumnos puedan desarrollar la tesis en formato de artículo científico (7).

Siendo la misión principal de una universidad la generación de conocimiento, la cual es medida en la actualidad por rankings de publicación internacional y teniendo en cuenta que no es ético dejar de mostrar los hallazgos de una investigación, esta baja tasa de publicación de tesis resulta preocupante.

Ante esto surgen algunas alternativas interesantes que ya están siendo aplicadas en algunas facultades de medicina que consiste el pedir, dentro de los requisitos para graduarse de Médico cirujano, el correo electrónico del editor de una revista indexada, aceptando la revisión por pares de su artículo científico (7). "La publicación desde el pregrado es una herramienta costo-eficaz que puede incrementar la visibilidad de la Universidad y estimular la investigación activa y constante en docentes y estudiantes" (7).

Enla era del internet, vale mucho más por su impacto, unas pocas páginas disponibles internacionalmente en la red, que un voluminoso empastado olvidado en la biblioteca de una facultad.

Entre las limitaciones del estudio está el no haber contado con todas las tesis de pregrado por diversos motivos.

En conclusión, las tesis de pregrado de la FMHUNPRG se caracterizaron por ser estudios descriptivos, realizados en población hospitalaria, de edad adulta, menos de la tercera parte de las tesis del periodo 2010-2014 tuvieron como tema una Prioridad de Investigación en Salud en el Perú, siendo la prioridad mortalidad materna la más estudiada.

La publicación de tesis es baja, llegándose a publicar en una revista indizada 1 de cada 25 tesis, aproximadamente. 


\section{REFERENCIAS BIBLIOGRÁFICAS}

1. Valle R, Salvador E. Análisis bibliométrico de las tesis de pregrado de la Facultad de Medicina de la Universidad Nacional Mayor de San Marcos. An Fac Med. 2009; 70(1):11-8.

2. Taype-Rondán A, Carbajal-Castro C, Arrunategui-Salas G, ChambiTorres J. Limitada publicación de tesis de pregrado en una facultad de medicina de Lima, Perú, 2000-2009. An Fac Med. 2012; 73(2):1537.

3. Arriola-Quiroz I, Curioso W, Cruz-Encarnacion M, Gayoso 0 . Characteristics and publication patterns of theses from a Peruvian medical school. Health Info Libr J. 2010; 27 (2):148-54.

4. Establecen normas mínimas de exigencia académica a fin de facilitar a los estudiantes universitarios la obtención de sus grados académicos que les permita acceder a puestos de trabajo. Decreto Legislativo Nro. 739. Boletin oficial de Normativa Nacional, (08 de noviembre de 1991).

5. Mayta-Tristán P, Mezones-Holguín E. Aclaración editorial. Rev Peru Med Exp Salud Publica. 2009; 26(3): 411-12.

6. Cabrera-Enríquez J, Cruzado-Mendoza C, Purizaca-Rosillo N, LópezSamanamú O, Lajo-Aurazo Y, Peña-Sánchez E. Factores asociados con el nivel de conocimientos y la actitud hacia la investigación en estudiantes de medicina en Perú, 2011. Rev Panam Salud Publica. 2013; 33(3): 166-73.

7. León Jiménez F. Pregrado en Medicina Humana: ¿Es posible graduarse publicando? Rev Med Hered. 2013; 24:175-177.

8. Alarco J, Aguirre-Cuadros E, Aliaga-Chávez Y, Álvarez-Andrade E. Factores asociados a la realización de tesis en pregrado de Medicina en una universidad pública del Perú. CIMEL. 2011; 15 (2): 66-70.

\section{Agradecimientos}

A la oficina del Centro de Investigación de la FMHUNPRG por facilitarnos la relación oficial de tesis y los in extensos no disponibles en la biblioteca.

\section{Presentaciones Previas}

Expuesto en el XXVIII Congreso Científico Nacional de Estudiantes de Medicina Humana.

\section{Fuentes de financiamiento}

Este artículo ha sido financiado por el autor.

\section{Conflictos de interés}

Los autores declaran no tener conflictos de interés.

\section{Correspondencia:}

Esteban Alberto Plasencia Dueñas

Correo: esteban_alberto147@hotmail.com Dirección: Calle Arica $N^{\circ} 1260$ - Chiclayo Teléfono: 942801942 